\title{
REPRESENTATIVIDAD A ESCALA REGIONAL DE UN INVENTARIO FLORÍSTICO DETALLADO DE UNA HECTÁREA EN LOS ANDES TROPICALES
}

\section{Regional scale representation based on a detailed floristic inventory in a one hectare plot in the tropical Andes}

\author{
Heriberto David-Higuita ${ }^{1} \&$ Esteban Álvarez-Dávila ${ }^{2}$
}

Higuita, H.D. \& Álvarez D, E. (2015). Representatividad a escala regional de un inventario florístico detallado de una hectárea en los Andes tropicales. Colombia Forestal, 18(2),207-224.

Recepción: 9 de septiembre de 2014

\section{RESUMEN}

Se reporta la composición florística de plantas vasculares y su distribución por hábitos de crecimiento en un bosque húmedo montano bajo en el norte de los Andes, con base en una parcela de 1 ha. Dentro de la parcela de 1 ha se colectaron muestras botánicas de manera intensiva y se identificaron 318 especies/morfoespecies, 82 familias (9 de las cuales pertenecen a la división Pteridophyta) y 173 géneros. Orchidaceae, Asteraceae, Rubiaceae, Melastomataceae y Piperaceae aportan el $44 \%$ del total de especies registradas en la parcela. Los géneros más diversos son Peperomia, Elaphoglossum, Mikania, Anthurium y Miconia. Se encontraron tres especies endémicas del departamento de Antioquia (Brunellia trianae, Meriania antioquiensis y Mikania fragrans). Las especies encontradas representan el $47 \%$, los géneros el $48 \%$ y las familias el $60 \%$ del total con respecto al Parque Arví, una reserva de 11 400 ha donde se localizó la parcela. Se encontraron 108 especies, 30 géneros y 1 familia no reportados previamente para el Parque Arví. El número de taxones comunes entre la parcela y el Parque fue de 144 especies, 142 géneros y 69 familias. La riqueza de especies/familia en la parcela explica el $89 \%$
Aprobación: 28 de abril de 2015

de la variación en la riqueza de especies/familia en al Parque Arví, y la riqueza de géneros el $75 \%$. Se concluye que los inventarios florísticos detallados en parcelas de área conocida aportan información representativa de la flora a nivel regional y permiten una aproximación rápida a su biodiversidad.

Palabras clave: andes tropicales, conteo total, muestreo intensivo, plantas no arbóreas, plantas vasculares.

\section{ABSTRACT}

Floristic composition of vascular plants and their distribution is reported for growth habits in a low montane rain forest $(2600 \mathrm{~m}$ and $2500 \mathrm{~mm} / \mathrm{yr}$ mean annual precipitation) in the northern Andes, based on a plot of 1 ha. The samples collected represented 318 species/morphospecies, 82 families (nine of which were pteridophytes) and 173 genera. $78 \%$ of the samples were identified to species. Groups with most species are pteridophytes (sensu lato), Orchidaceae, Asteraceae, Rubiaceae, Melastomataceae and Piperaceae, that represented $44 \%$ of all species recorded. The most diverse genera are Peperomia, Elaphoglossum, Mikania, Anthurium, and Miconia. 
Three species endemic to Antioquia (Brunellia trianae, Meriania antioquiensis and Mikania fragrans) were found. The individuals found in the plot represent $47 \%$ of the species, $48 \%$ of the genera and $60 \%$ of the families found in Arví Park, a reserve of 11400 hectares in which the plot was located. The plot had 108 species, 30 genera and one family not previously reported for Arvi Park. The number of common taxa between the plot and the Arví Park was 144 species, 142 genera and 69 families. Species richness/family in the plot accounts for $89 \%$ of the variation of species richness/family in the Arví Park, and $75 \%$ of the generic richness. We conclude that detailed floristic inventory of known area plots provide information representative of the regional flora and permit a rapid approximation of its biodiversity.

Keywords: tropical Andes, total count, intensive sampling, non-woody plants, vascular plants.

\section{INTRODUCCIÓN}

Los Andes tropicales constituyen uno de los principales centros de diversidad de plantas del mundo, con alta presencia de endemismos (Young et al., 2002), altas tasas de especiación (Madriñán et al., 2013) y al mismo tiempo con mayores niveles de amenaza (Myers et al., 2000). Sin embargo, el conocimiento de la flora de los Andes tiene muchos vacíos de información, particularmente en Colombia (Jorgensen et al.,2012), por lo que es importante desarrollar estudios que contribuyan a su conocimiento.

Normalmente, los inventarios florísticos de una región se construyen mediante la colección de muestras botánicas en diferentes sitios, tratando de abarcar la mayor parte de la variación espacial/ ambiental y haciendo énfasis en la colecta de individuos que se encuentran en estado reproductivo; generalmente estos inventarios requieren la inversión de una gran cantidad de recursos de tiempo, personal y dinero (Phillips et al., 2003). Por tal razón, desde hace décadas se han propuesto técnicas de inventarios rápidos, bajo el supuesto de que permiten tener una idea de la composición y diversidad de un sitio, un paisaje o una región, con una baja inversión de recursos (Jayakumar et al., 2011; Gordon \& Newton, 2006). Los métodos rápidos más comunes consideran grupos indicadores bien sea a nivel taxonómico, funcional o individuos a partir de cierto tamaño (Phillips et al., 2003; Higgins \& Ruokolainen, 2004).
Adicionalmente, algunos investigadores han realizado inventarios detallados de plantas vasculares en parcelas de área conocida, considerando todos los hábitos de crecimiento: árboles, arbustos, lianas, epífitas, etc. (Linares-Palomino et al., 2009). Todos estos estudios confirman que en áreas tan pequeñas como $100 \mathrm{~m}^{2}$ (Whitmore et al., 1985), $1000 \mathrm{~m}^{2}$ (Gentry \& Dodson, 1987) y 1 ha (Valencia et al., 1994) se pueden encontrar cientos de especies que cuando se comparan con otros listados publicados a nivel regional, representan una gran proporción del total de especies registradas para una región, y que dan como resultado adicional una alta similaridad florística a nivel de géneros y familias (Duivenvoorden, 1994) e incluso hasta especies (Kelly et al., 1994). No obstante, una restricción importante de estas comparaciones es que se hacen generalmente sin confrontar las colecciones botánicas directamente en herbario; lo cierto es que esta aproximación es válida para familias, e incluso para géneros, pero presenta problemas cuando se hace a nivel de especies, ya que cuando se usan nombres y no muestras botánicas, se presenta una gran incertidumbre en las comparaciones; es bien conocido que entre herbarios existen diferencias en el nivel de curaduría de las colecciones, así como sesgos relacionados con la experiencia de los botánicos a cargo de la identificación (Lópes \& Duque, 2010). Adicionalmente, la comparación a nivel de géneros o familias asume que los patrones 
resultantes son similares a los que se obtendrían con las especies, lo que ha sido verificado en estudios en bosques tropicales de tierras bajas ( $\mathrm{Hi}-$ ggins \& Ruokolainen, 2004; Cayuela et al., 2011), pero no en los bosques de montaña de los Andes tropicales (Lópes \& Duque, 2010).

En el departamento de Antioquia, localizado en el extremo norte de los Andes en Colombia, y con un área total de $66000 \mathrm{~km}^{2}$, se han reportado cerca de 8000 especies de plantas vasculares, por lo que es una de las regiones mejor exploradas de Colombia (Idarraga et al., 2011; Arbeláez-Cortés, 2013). En particular, el Parque Regional Arví, un área protegida localizada en el centro de Antioquia, con altitudes entre 2200 y 2800 m de altitud, alberga remanentes importantes de bosques andinos y corresponde a la región con mayor número de estudios florísticos del departamento. Este parque cubre alrededor de 11500 ha, donde se han registrado hasta la fecha 812 especies de plantas vasculares, 40 de ellas endémicas del departamento de Antioquia (Toro, 2009). En este sentido, este sitio representa una gran oportunidad para poner a prueba la hipótesis de que inventarios florísticos intensivos en parcelas de área conocida pueden dar una idea apropiada de la riqueza y composición de especies a escalas mayores.

En este trabajo se realizó un inventario detallado de plantas vasculares dentro de 1 ha en un bosque montano del Parque Arví, incluyendo todos los hábitos de crecimiento y plantas de todos los tamaños con el objetivo de caracterizar la composición florística de la parcela y evaluar su representatividad en relación con la flora del Parque Arví (Kelly et al., 1994; Valencia et al., 1994; Linares-Palomino et al., 2009). Concretamente se trató de responder a la siguiente pregunta: ¿Qué tan representativa es la composición y riqueza de especies de una parcela de 1 ha a escala regional?

\section{METODOLOGÍA}

\section{Área de estudio}

El estudio se realizó en la Reserva Natural Montevivo, la cual ocupa una área aproximada de 50 ha, forma parte del Parque Regional Arví e integra territorios de los municipios de Medellín y Guarne, en el departamento de Antioquia, a una altura entre 2500 - $2680 \mathrm{~m}$ de altitud en la Cordillera Central de los Andes de Colombia (Figura 1). La Reserva está cubierta en su mayor parte por bosque nativo en diferentes estadíos sucesionales con algunos remanentes de plantaciones forestales. Las coordenadas de la Reserva son: $6.21^{\circ}$ de latitud norte y $75.49^{\circ}$ de longitud oeste.

De acuerdo con el sistema de clasificación de zonas de vida establecido por Holdridge (1982), el área corresponde a la zona de vida bosque húmedo montano bajo (bh-MB) (Espinal, 2011), con una temperatura promedio de $17^{\circ} \mathrm{C}$ y temperaturas máximas y mínimas de $20^{\circ} \mathrm{C}$ y $5^{\circ} \mathrm{C}$ respectivamente; la precipitación media anual es de $1948 \mathrm{~mm}$, en todos los meses se presentan más de $100 \mathrm{~mm}$ de lluvia y la humedad relativa promedio anual es del $82 \%$. El relieve de la parcela está formado por pequeñas colinas con pendientes entre $25^{\circ}-45^{\circ}$. Sus suelos son ácidos, de textura arcillosa, con cenizas volcánicas tipo andisol, con alto contenido de materia orgánica (Toro, 2000). El paisaje alrededor de la Reserva consiste en fragmentos de bosque rodeados por una matriz de cultivos y potreros (Duque et al., 2013).

\section{Métodos}

Recolección de datos de campo. En el 2003 se estableció una parcela permanente de una hectárea $(100 \times 100 \mathrm{~m})$, el $50 \%$ de la cual corresponde a bosque primario, y el $50 \%$ restante a un bosque secundario avanzado (> 50 años); en la parcela se colectaron muestras botánicas de todas las plantas 
con DAP $>=5 \mathrm{~cm}$. Adicionalmente, en una muestra de 25 cuadrantes de $100 \mathrm{~m}^{2}$ dentro de la parcela se hicieron colecciones de todas las especies presentes, incluyendo árboles, lianas, hemiepífitas, helechos y hierbas terrestres, orquídeas terrestres, bromelias y aráceas; estas últimas incluyeron muestreos en las copas de los árboles (Figura 1).
Trabajo de herbario. El material vegetal colectado fue determinado con la ayuda de bibliografía especializada, claves, monografías, comparación con colecciones de los herbarios HUA (Universidad de Antioquia) y JAUM (Jardín Botánico de Medellín), la base de datos del Missouri Botanical Garden y con la colaboración de
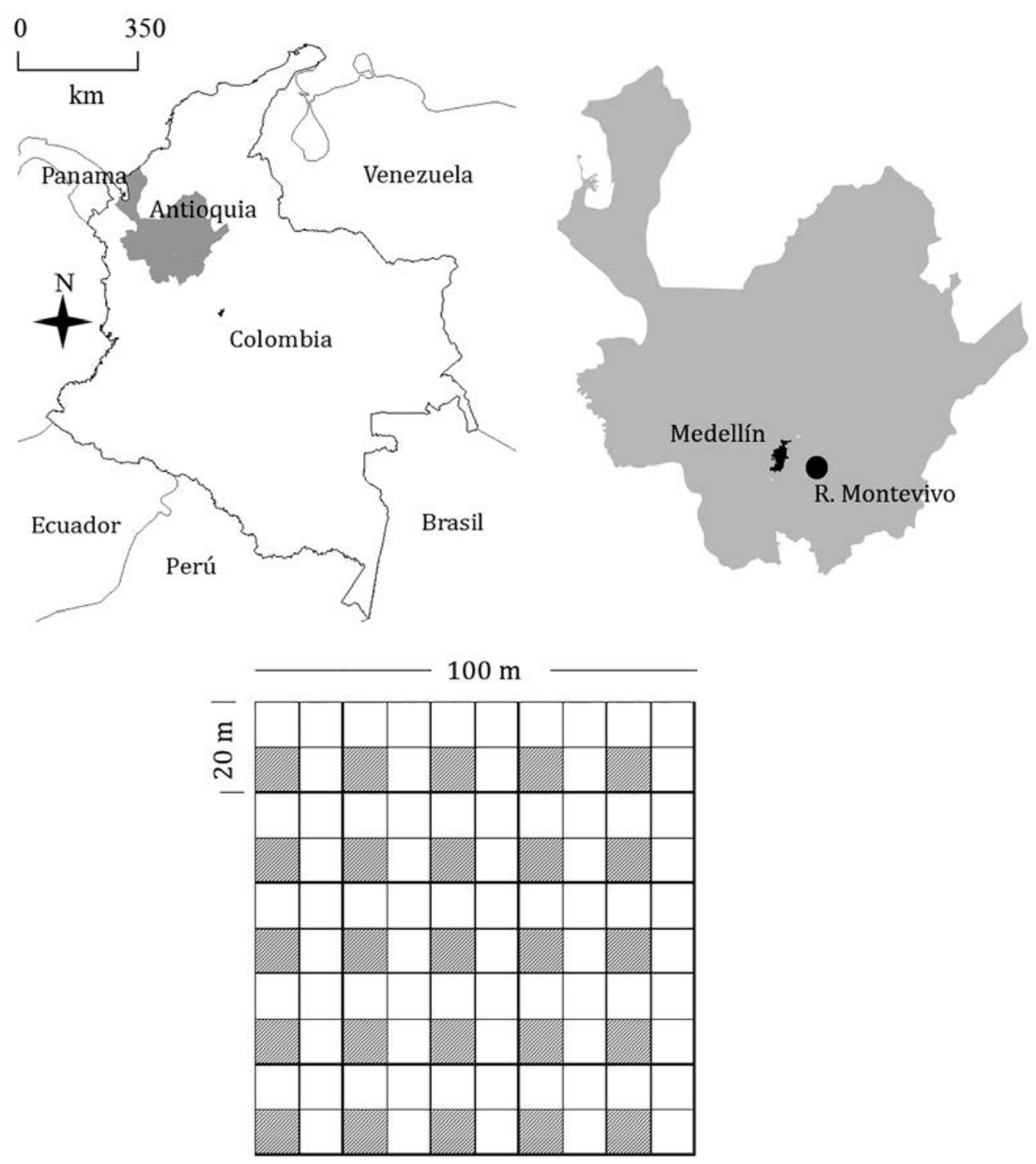

Figura 1. Localización del área de estudio en la Reserva Montevivo y tipo de parcela empleado para el muestreo. En toda la parcela de $100 \times 100 \mathrm{~m}$ se hizo el muestreo para plantas con DAP $\geq 5 \mathrm{~cm}$, y en los 25 cuadrantes de 10 $\times 10 \mathrm{~m}$ (cuadrículas grises) se hizo el muestreo de toda la vegetación con $\mathrm{DAP}<5 \mathrm{~cm}$, incluyendo epífitas. 
especialistas en algunos grupos o familias botánicas. Las muestras botánicas fueron depositadas en los herbarios mencionados bajo la numeración del primer autor (Heriberto David Higuita, $\mathrm{HDH})$. A cada especie colectada se le asignó un "hábito de crecimiento" de acuerdo con observaciones de campo. Para las especies que presentaban varios hábitos (por ejemplo, es común encontrar muchas herbáceas a veces como terrestres o como epífitas, o lianas que se encuentra como arbustos), se definió uno solo de acuerdo con su predominancia en campo. Las categorías se asignaron de acuerdo con lo propuesto por Londoño et al. (1995), los cuales consideran cinco categorías: árboles $(\mathrm{Ar})$, arbustos $(\mathrm{Ab})$, escandentes leñosos (Li), escandentes herbáceos (Eh), hierbas terrestres $(\mathrm{Ht})$ y epífitas (Ep).

Para la nomenclatura de los nombres científicos se usaron las bases de datos más actualizadas, aplicando el siguiente procedimiento: 1) La lista de especies obtenida a partir del trabajo de herbario fue ingresada en la aplicación TNR-Taxonomic Name Resolution (Boyle et al., 2013) que permite verificar la forma correcta de escribir los nombres y corregir las sinonimias que persisten en los herbarios consultados (HUA y JAUM); esta aplicación permite la consulta simultánea de hasta 500 nombres científicos en las bases de datos de plantas más importantes del mundo como The International Plant Names Index (IPNI, 2012) y Tropicos (Garden, 2003). 2) Las inconsistencias no resueltas con el usos de $T N R$ fueron consultadas posteriormente en la base de datos The Plant List (TPL, 2010).

Análisis de los datos. Para evaluar la representatividad del inventario de la Reserva Montevivo en relación con la flórula del Parque Arví, las muestras colectadas fueron comparadas con las colecciones botánicas depositadas en los herbarios HUA y JAUM para la región (Natura \& Holos,
2001; Toro, 2000; 2009). Con esta información se contrastó la riqueza de especies, géneros y familias entre los dos muestreos y la semejanza florística en diferentes niveles taxonómicos. Adicionalmente, se realizó un análisis de regresión lineal simple para evaluar si la riqueza de especies por familias y géneros estaba correlacionada entre el inventario realizado para este trabajo y el del Parque Arví.

\section{RESULTADOS}

\section{Composición florística de la parcela de Montevivo}

En total, para la parcela de 1 ha se colectaron 318 especies de plantas vasculares, de las cuales 270 especies son angiospermas y 49 especies son Pteridophytas (Anexo 1). Del total de especies encontradas, $318(99.7 \%)$ están determinadas hasta familia, 316 (99\%) hasta género, 253 (79.3\%) hasta especie, y una morfoespecie $(0.3 \%)$ permanece indeterminada. En las angiospermas se encontraron 71 familias y 148 géneros, y en las Pteridophytas se encontraron 49 especies y 23 géneros. El número de especies encontradas en cada familia y categorías de hábitos de crecimiento se presentan en la Tabla 1. En el listado se reportan tres especies consideradas como endémicas para Antioquia: Brunellia trianae, Meriania antioquiensis y Mikania fragrans.

31 familias (43.7\% del total encontradas) están representadas por una sola especie y solo 15 familias (20.8\% del total) contienen el 68\% del número total de especies, siendo las Pteridophytas (sensu lato), Orchidaceae, Asteraceae, Rubiaceae, Melastomataceae, Piperaceae, Ericaceae y Solanaceae las familias que contienen más de 10 especies. 
Tabla 1. Número de especies de plantas vasculares por familia en un 1 ha de bosque andino en la Reserva Montevivo.

\begin{tabular}{|c|c|c|c|c|c|c|c|c|c|c|c|c|c|c|c|}
\hline Familia & $\frac{\mathscr{E}}{\frac{\mathscr{U}}{\delta}}$ & $\frac{\stackrel{0}{0}}{\frac{0}{2}} \frac{0}{\frac{0}{2}}$ & 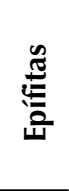 & 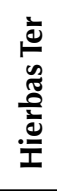 & 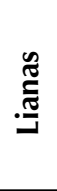 & 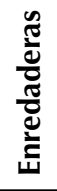 & $\underset{\mathbb{0}}{0}$ & Familia & $\frac{\frac{\mathscr{U}}{0}}{\frac{0}{2}}$ & $\frac{\stackrel{n}{0}}{\frac{0}{2}}$ & 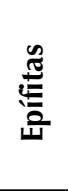 & 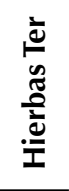 & 胥 & 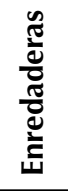 & $\frac{\bar{\pi}}{0}$ \\
\hline MAGNOLIOPHYTA & 59 & 84 & 32 & 36 & 24 & 34 & 269 & Brunelliaceae & 1 & & & & & & 1 \\
\hline Orchidaceae & & & 12 & 11 & & & 23 & Chrysobalanaceae & & 1 & & & & & 1 \\
\hline Asteraceae & & 2 & & 1 & & 17 & 20 & Dichapetalaceae & & 1 & & & & & 1 \\
\hline Rubiaceae & & 12 & & 3 & & 3 & 18 & Dioscoreaceae & & & & & & 1 & 1 \\
\hline Melastomataceae & 6 & 7 & 1 & & 1 & & 15 & Gentianaceae & & 1 & & & & & 1 \\
\hline Piperaceae & & 6 & 6 & 3 & & & 15 & Grossulariaceae & 1 & & & & & & 1 \\
\hline Ericaceae & & 6 & 1 & & 5 & & 12 & Hydrangeaceae & & & & & 1 & & 1 \\
\hline Solanaceae & & 5 & & & 5 & & 10 & Indet & & & & & 1 & & 1 \\
\hline Bromeliaceae & & & 8 & 1 & & & 9 & Lacistemataceae & 1 & & & & & & 1 \\
\hline Araceae & & & 1 & 7 & & & 8 & Lecythidaceae & & 1 & & & & & 1 \\
\hline Lauraceae & 2 & 6 & & & & & 8 & Loranthaceae & & & & 1 & & & 1 \\
\hline Myrtaceae & 4 & 4 & & & & & 8 & Meliaceae & 1 & & & & & & 1 \\
\hline Araliaceae & 4 & 2 & & & 1 & & 7 & Moraceae & & 1 & & & & & 1 \\
\hline Clusiaceae & 6 & & 1 & & & & 7 & Myricaceae & 1 & & & & & & 1 \\
\hline Euphorbiaceae & 6 & 1 & & & & & 7 & Poaceae & & & & 1 & & & 1 \\
\hline Caprifoliaceae & 3 & & 1 & & & 1 & 5 & Polygonaceae & & & & & 1 & & 1 \\
\hline Cyperaceae & & & & 5 & & & 5 & Ranunculaceae & & & & & 1 & & 1 \\
\hline Primulaceae & 2 & 3 & & & & & 5 & Rhamnaceae & & 1 & & & & & 1 \\
\hline Rosaceae & 1 & 2 & & & & 2 & 5 & Salicaceae & & 1 & & & & & 1 \\
\hline Campanulaceae & & 3 & & & & 1 & 4 & Simaroubaceae & & 1 & & & & & 1 \\
\hline Polygalaceae & & 2 & & & 1 & 1 & 4 & Staphyleaceae & 1 & & & & & & 1 \\
\hline Alstroemeriaceae & & & & & & 3 & 3 & Styracaceae & & 1 & & & & & 1 \\
\hline Apocynaceae & & & & & & 3 & 3 & Thymelaeaceae & & 1 & & & & & 1 \\
\hline Aquifoliaceae & 2 & 1 & & & & & 3 & Urticaceae & & & & 1 & & & 1 \\
\hline Cunoniaceae & 2 & 1 & & & & & 3 & Violaceae & & & & 1 & & & 1 \\
\hline Gesneriaceae & & 2 & 1 & & & & 3 & Vitaceae & & & & & 1 & & 1 \\
\hline Acanthaceae & & 2 & & & & & 2 & Winteraceae & 1 & & & & & & 1 \\
\hline Anacardiaceae & 1 & 1 & & & & & 2 & PTERIDOPHYTA & 3 & & 24 & 21 & & 1 & 49 \\
\hline Boraginaceae & 1 & 1 & & & & & 2 & Dryopteridaceae & & & 6 & 6 & & & 12 \\
\hline Celastraceae & & 1 & & & 1 & & 2 & Polypodiaceae & & & 11 & & & & 11 \\
\hline Chloranthaceae & 2 & & & & & & 2 & Blechnaceae & & & 1 & 2 & & & 3 \\
\hline Clethraceae & 2 & & & & & & 2 & Cyatheaceae & 3 & & & & & & 3 \\
\hline Malpighiaceae & & & & & 2 & & 2 & Hymenophyllaceae & & & 3 & & & & 3 \\
\hline Monimiaceae & & 1 & & & 1 & & 2 & Lycopodiaceae & & & & 3 & & & 3 \\
\hline Passifloraceae & & & & & & 2 & 2 & Pteridaceae & & & & 2 & & 1 & 3 \\
\hline Proteaceae & 2 & & & & & & 2 & Aspleniaceae & & & 2 & & & & 2 \\
\hline Rutaceae & 2 & & & & & & 2 & Dennstaedtiaceae & & & & 2 & & & 2 \\
\hline Sapindaceae & 2 & & & & & & 2 & Marattiaceae & & & & 2 & & & 2 \\
\hline Smilacaceae & & & & & 2 & & 2 & Thelypteridaceae & & & & 2 & & & 2 \\
\hline Theaceae & 1 & 1 & & & & & 2 & Equisetaceae & & & & 1 & & & 1 \\
\hline Verbenaceae & 1 & 1 & & & & & 2 & Gleicheniaceae & & & & 1 & & & 1 \\
\hline Actinidaceae & & 1 & & & & & 1 & Polypodiaceae & & & 1 & & & & 1 \\
\hline Balanophoraceae & & & & 1 & & & 1 & Total general & 62 & 84 & 56 & 57 & 24 & 35 & 318 \\
\hline
\end{tabular}


La Figura 2a muestra la proporción en que cada forma de crecimiento aporta a la riqueza de especies. Los árboles y arbustos contienen el mayor número de especies, seguidos por las hierbas terrestres y las epífitas. El componente no arbóreo (hierbas terrestres, epífitas y hemiepífitas herbáceas y escandentes) representa el $54.3 \%$ del total de especies en la parcela; si se excluyen los escandentes leñosos (o lianas, con 25 especies), las plantas no leñosas corresponde al $46.2 \%$ del total. La Figura $2 \mathrm{~b}$ muestra los géneros con mayor número de especies; estos 16 géneros contienen $29.2 \%$ del total de especies, y son principalmente no leñosos (Elaphoglossum, Peperomia, Epidendrum, Polypodium, Pleurothallis, Anthurium, Rhynchospora, Mikania, Pentacalia) o con especies en ambas grupos funcionales (Clusia, Solanum, Monnina, Miconia) y solo tres géneros con especies exclusivamente leñosas (Piper, Palicourea, Psychotria). En la Tabla 2 se presentan los géneros por hábito de crecimiento y el respectivo número de especies.
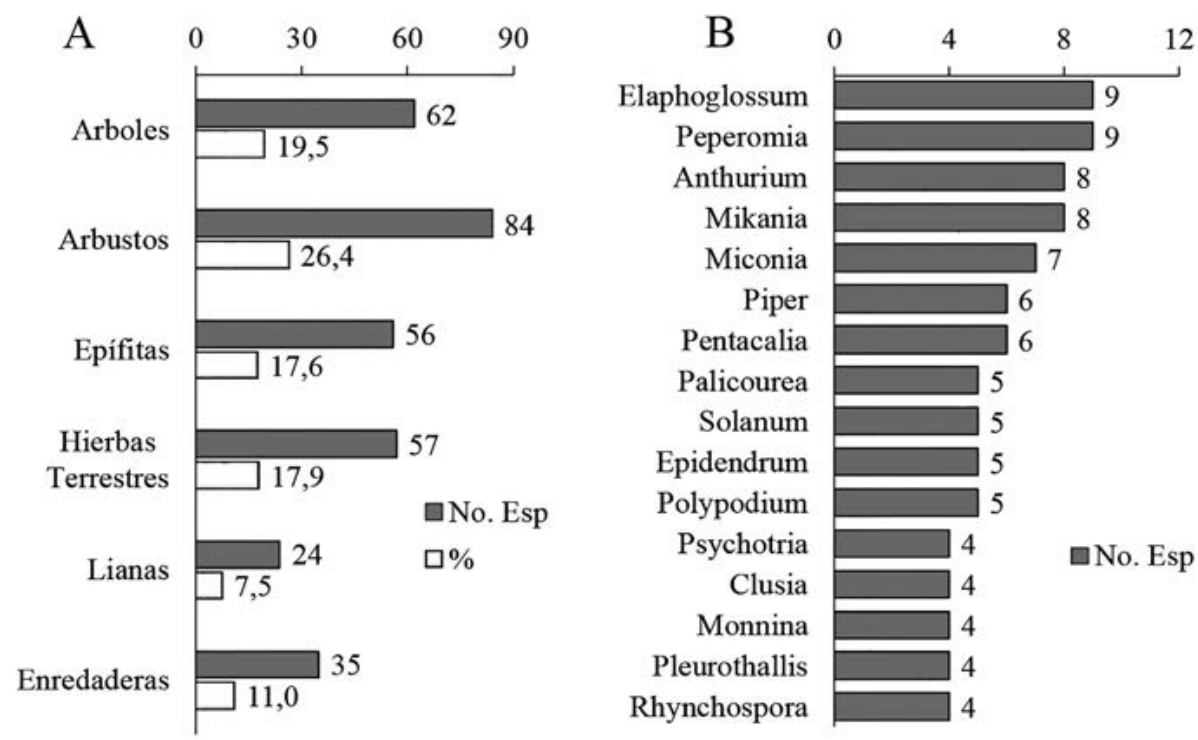

Figura 2. (A) Número de especies para diferentes hábitos de crecimiento en 1 ha de bosque andino en el oriente antioqueño, Colombia. (B) Géneros con mayor riqueza de especies. 


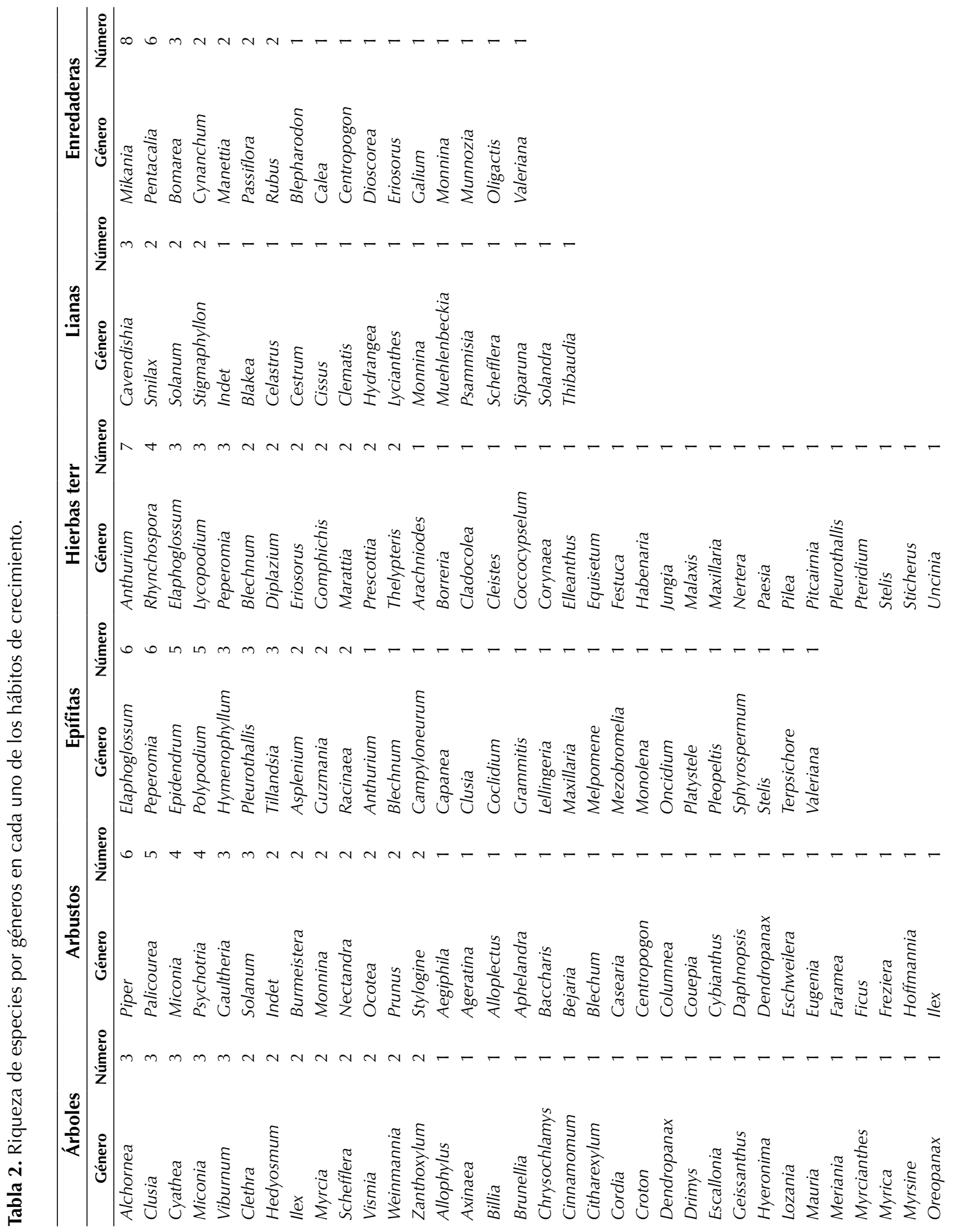




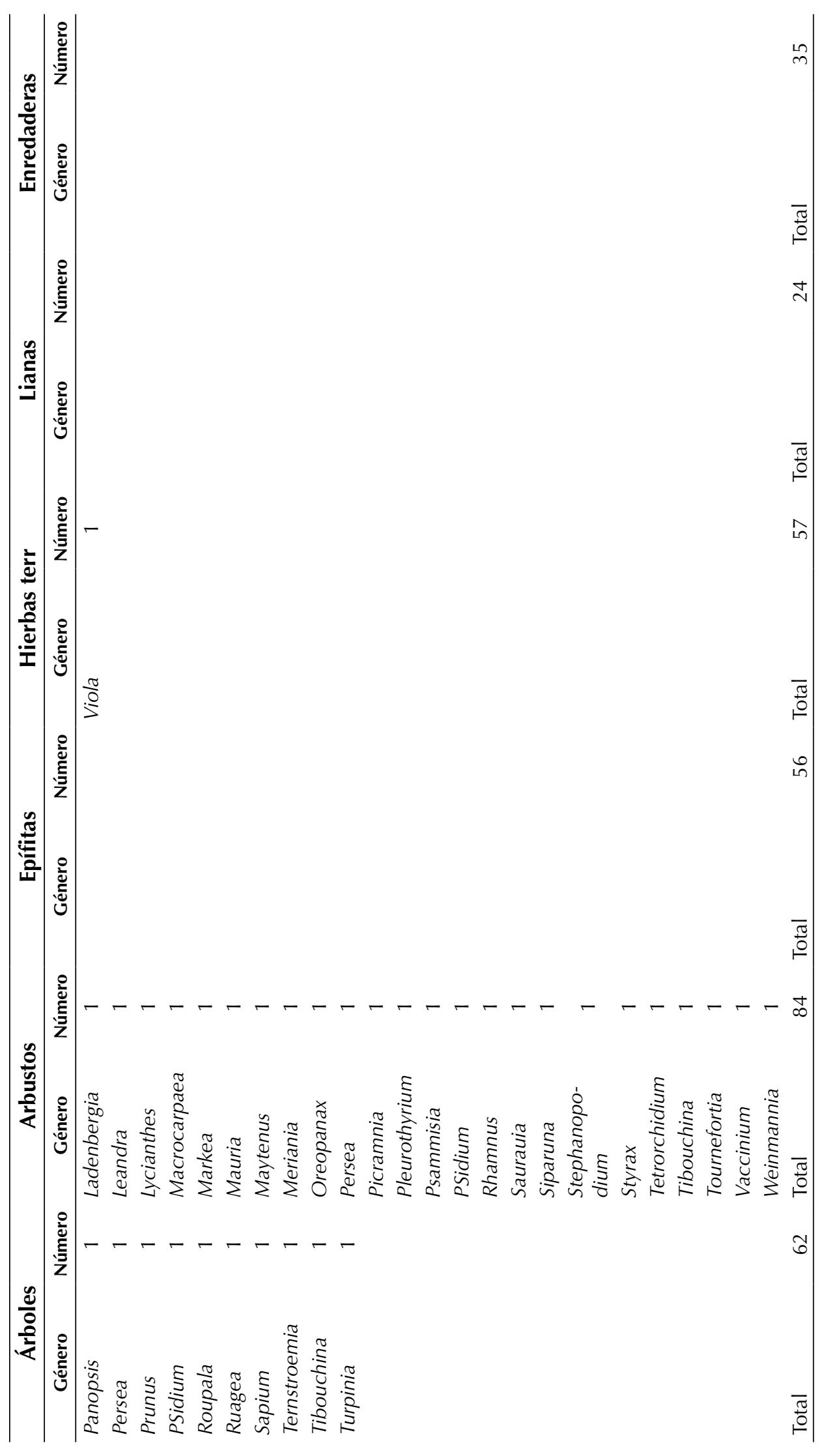




\section{Representatividad de la flora de Montevivo con relación al Parque Arví}

En el estudio original de la Flora del Parque Arví (Toro, 2000; Natura \& Holos, 2001) se reportaron 615 morfoespecies, 482 de las cuales estuvieron identificadas hasta especie (genero + epíteto). La

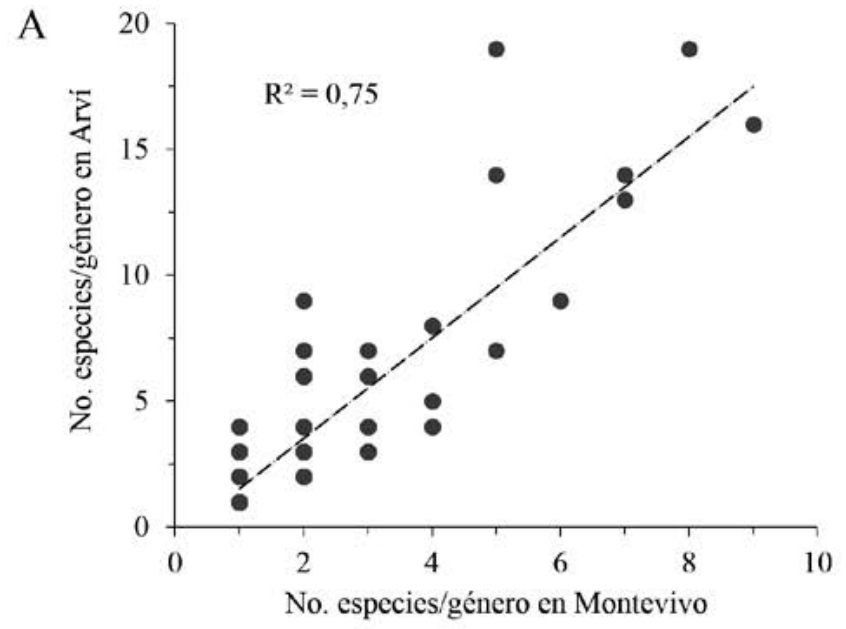

comparación de estos resultados con el listado florístico de la parcela de 1 ha en la Reserva Montevivo indica que el inventario desarrollado añade a la flora del Parque Arví una nueva familia (Balanophoraceae), 30 géneros y 108 especies. En total se encontraron 79 familias, 142 géneros y 144 especies compartidas (Tabla 3).

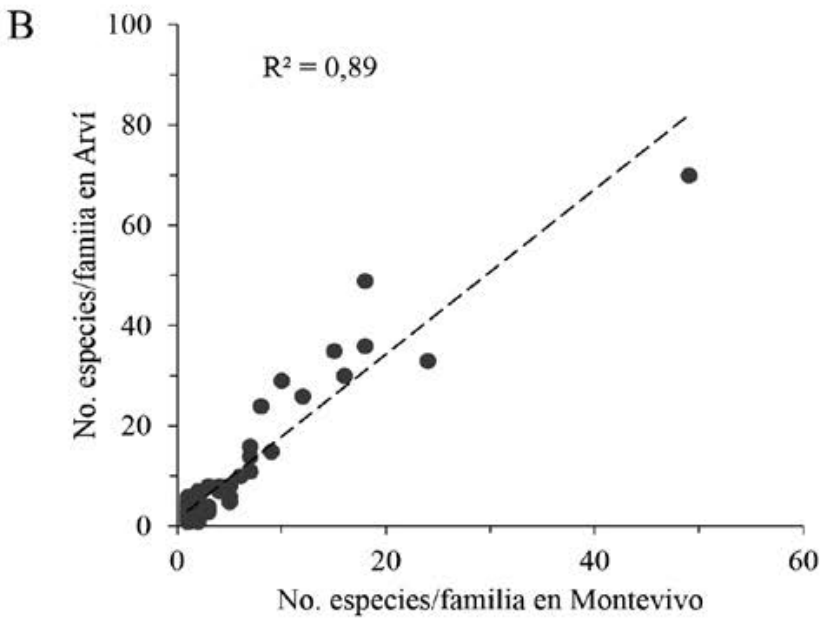

Figura 3. Los circulos muestran la riqueza de especies de (A) géneros compartidos y (B) familias compartidas entre la parcela de Montevivo (este estudio) y la flórula del Parque Arví (Natura \& Holos 2011). La línea corresponde a una regresión lineal simple; en ambos casos la regresión fue altamente significativa $(p<0.001)$.

Tabla 3. Comparación de la composición florística entre la Reserva Montevivo (este estudio) y la flórula del Parque Arví (Corantioquia, 2001), indicando el nivel de identificación taxonómica.

\begin{tabular}{|c|c|c|c|c|c|c|c|c|}
\hline \multirow[b]{2}{*}{ División } & \multicolumn{4}{|c|}{ Parcela 1 ha Reserva Montevivo } & \multicolumn{4}{|c|}{ Flórula del Parque Arví } \\
\hline & $\begin{array}{c}\text { Hasta } \\
\text { especie }\end{array}$ & $\begin{array}{l}\text { Hasta } \\
\text { género }\end{array}$ & $\begin{array}{c}\text { Hasta } \\
\text { familia }\end{array}$ & Total & $\begin{array}{c}\text { Hasta } \\
\text { especie }\end{array}$ & $\begin{array}{c}\text { Hasta } \\
\text { género }\end{array}$ & $\begin{array}{c}\text { Hasta } \\
\text { familia }\end{array}$ & Total \\
\hline Magnoliophyta & 229 & 62 & 1 & 292 & 482 & 121 & 12 & 615 \\
\hline Pteridophyta & 20 & 6 & & 26 & 41 & 19 & & 60 \\
\hline Total & 249 & 68 & & 318 & 523 & 140 & 12 & 675 \\
\hline Número de géneros & \multicolumn{4}{|c|}{ Montevivo } & \multicolumn{4}{|c|}{ Parque Arví } \\
\hline Magnoliophyta & \multicolumn{4}{|c|}{162} & \multicolumn{4}{|c|}{328} \\
\hline Pteridophyta & \multicolumn{4}{|c|}{11} & \multicolumn{4}{|c|}{35} \\
\hline Total & \multicolumn{4}{|c|}{173} & \multicolumn{4}{|c|}{363} \\
\hline Número de familias & \multicolumn{4}{|c|}{ Montevivo } & \multicolumn{4}{|c|}{ Parque Arví } \\
\hline Magnoliophyta & \multicolumn{4}{|c|}{73} & \multicolumn{4}{|c|}{119} \\
\hline Pteridophyta & \multicolumn{4}{|c|}{9} & \multicolumn{4}{|c|}{18} \\
\hline Total & \multicolumn{4}{|c|}{82} & \multicolumn{4}{|c|}{137} \\
\hline
\end{tabular}


La comparación de la riqueza de especies por género (Figura 3A) indica que existe una alta semejanza entre las dos floras, considerando que el $75 \%$ de la variación regional es explicada por la riqueza de especies en Montevivo. No obstante, 12 géneros tienen mayor riqueza de especies en Montevivo que en Arví: Elaphoglossum, Mikania y Pentacalia (4 especies más en Arví), Prunus (2 especies más en Arví), mientras que con una especie más se registran, Cynanchum, Diplazium, Lycianthes, Mauria, Prescottia, Racinaea, Stigmaphyllon y Stylogyne. En el caso de las familias (Figura 3B), la riqueza de especies fue similar para todas las familias comunes $(89 \%$ de la variación en la riqueza regional de especies/familia con base en nuestro inventario), excepto para Poaceae, que tiene 22 especies en Arví, pero solo una en Montevivo.

\section{DISCUSIÓN}

\section{Representatividad del inventario a escala regional}

La composición a nivel de familias para la parcela de Montevivo presenta muchos de los elementos típicos reportados para bosques andinos entre 2000 - 3000 m de altitud tanto para árboles, plantas leñosas y demás hábitos de crecimiento (Gentry, 1995; Kelly et al., 1994). Adicionalmente se encontró que existe una alta proporción de géneros comunes entre el estudio realizado y los reportados para el Parque Arví, así como una alta correlación entre el número de especies por género entre los dos bosques, igual que para familias; esto quiere decir que en general los géneros y familias con mayor riqueza de especies a escala regional estuvieron igualmente bien representados en la parcela de 1 ha; un caso particular como el de la familia Poaceae, para la cual solo se encontró una especie en Montevivo y 22 en el Parque Arví, puede explicarse debido a que las especies de esta familia en general prefieren áreas abiertas, lo que hace que estén subrepresentadas en la parcela de Montevivo. Las 318 especies encontradas en la parcela de la Reserva Montevivo, representan una porción importante $(40 \%$ del total) de las especies reportadas para el Parque Arví, considerando la diferencia en área entre los dos sitios. Estos resultados son evidencias de que la flora encontrada en una parcela de 1 ha en Montevivo es representativa de la composición a escala regional.

La alta riqueza de especies que se puede encontrar en parcelas pequeñas $(<=1 \mathrm{ha})$ cuando se considera el total de las plantas vasculares, y no sólo árboles, fue inicialmente mostrada por Gentry \& Dodson (1987) en un estudio en el occidente del Ecuador donde se reportaron hasta 365 especies de plantas vasculares en una parcela de 0.1 ha, de las cuales apenas 32 especies corresponden a árboles grandes (DAP $\geq 10 \mathrm{~cm}$ ). En el Chocó colombiano se reportaron 442 y 970 especies en una muestra de 0.1 ha y 0.9 ha, respectivamente (Galeano et al., 1998); menos del $10 \%$ de las especies se reportan en este estudio para los árboles grandes y menos del 30\% para las plantas leñosas con DAP $>=2.5 \mathrm{~cm}$. Otros estudios realizados en parcelas de 1 ha reportan resultados similares de alta riqueza de especies: por ejemplo, Balslev et al. (1998) encontraron 942 especies de plantas vasculares en 88 familias (con los árboles de todos los tamaños aportando el $50 \%$ de las especies) en las tierras bajas de la amazonia ecuatoriana. Linares-Palomino et al. (2009) reportaron entre 297 a 382 especies en 60-75 familias/ha para tres bosques en el Refugio Los Volcanes, en Bolivia, a 1000 m de altitud, el $24 \%$ de las cuales eran árboles. Otro estudio en la Amazonia colombiana reportan un máximo de 313 especies en parcelas de 0.1 ha, con una alta proporción de árboles del 50\% del total de especies (Duivenvoorden, 1994). El único estudio en un bosque montano similar al aquí presentado fue realizado por Kelly et al., (1994) en los Andes de Venezuela, en una parcela de 1,5 ha, donde se encontraron 219 especies, de las cuales solo 52 fueron árboles. 
En todos estos estudios se indica que el número de especies encontradas en las parcelas representa una parte importante del total reportado a escala regional. Adicionalmente, la proporción deespecies no arbóreas muestra un patrón consistente, y en general se puede afirmar que son un componente importante de los bosques neotropicales. Este patrón es evidentemente ignorado cuando solo se consideran únicamente los árboles, o las plantas leñosas, en los inventarios en parcelas; en particular, los árboles grandes con DAP $\geq 10 \mathrm{~cm}$ representan una proporción de especies inferior al $10 \%$ del total en la mayoría de los casos (LinaresPalomino et al., 2009).

Se debe resaltar que, a pesar de la baja diversidad y riqueza de especies que se obtienen a partir de inventarios donde se consideran solo árboles con DAP $\geq 10 \mathrm{~cm}$, estos son utilizados con frecuencia para caracterizar tipos de vegetación en diferentes escalas, con la justificación de que son el elemento estructural más importante del bosque (Duivenvoorden \& Lips, 1995; Ter Steege et al., 2000) Si bien es cierto que los árboles pueden ser suficientes para identificar los principales tipos de bosque, y estos correlacionan en ciertos casos con la diversidad de otros grupos funcionales (Duivenvoorden, 1994; Ruokolainen et al., 2007) varios estudios muestran que no existe un patrón consistente (Williams-Linera et al., 2005; Tchouto et al., 2006; Linares-Palomino et al., 2009). Comparar la composición florística de muestras obtenidas en parcelas con flórulas realizadas en áreas mayores tiene ciertas restricciones: en primer lugar, las muestras de las parcelas son incompletas y ciertos grupos funcionales (como especialistas de áreas abiertas o epífitas) pueden estar subrepresentados (Duivenvoorden, 1994). Además, la categorización dentro de "morfoespecies" que es necesaria en las colecciones en parcelas puede influir también en la comparación, especialmente cuando estas representan una gran parte de las identificaciones, como en Gentry (1982), con el $44 \%$ de las especies identificadas completamente, o en Duivenvoorden (1994), con el 50\% de las especies identificadas completamente. No obstante, en este estudio, para la parcela de la Reserva Montevivo, la proporción de especies plenamente identificadas fue relativamente alto $(79 \%)$ y similar al porcentaje de especies identificadas en la flórula del Parque Arví (76\%).

Por otra parte, los inventarios en parcelas facilitan el hallazgo de nuevas especies que no son visibles mediante los métodos tradicionales de hacer recorridos a lo largo del bosque. Esto es evidente cuando se considera en el caso del presente trabajo el gran número de reportes nuevos de especies y géneros (e incluso una familia) que no habían sido encontrados en los estudios realizados previamente en el Parque Arví a lo largo de un período de diez años. Otros estudios en parcelas han logrado también hallazgos botánicos importantes; por ejemplo, el primer reporte para Suramérica del género Sapranthus (Vélez-Arango \& Cogollo-Pacheco, 2007) y el hallazgo de un género nuevo y segundo reporte de la familia para el neotrópico de la familia Dipterocarpaceae (Pseudomonotes tropenbosii; Londoño et al., 1995), para mencionar solo un par de casos destacados, fueron posibles gracias al esfuerzo de colecciones intensivas en parcelas.

\section{Importancia para la conservación}

Un gran número de publicaciones de la última década ubica con certeza los principales centros de diversidad de plantas vasculares a escala mundial (por ejemplo, Kier et al., 2009) y existe un consenso entre la comunidad científica sobre el conjunto mínimo global de áreas irremplazables por su biodiversidad, donde la conservación sería más eficaz (Brooks et al., 2006). Sin embargo, en muchas de estas áreas, como en el norte de los Andes, el estado del conocimiento florístico es a menudo sesgado, tanto taxonómica como geográficamente (Jorgensen et al., 2012); además, el impacto de las actividades humanas es creciente y la pérdida de especies y ecosistemas probablemente supere ampliamente el ritmo de su documentación. 
En este sentido, el mejoramiento de los "métodos rápidos" para el inventario de la flora es una necesidad urgente.

El departamento de Antioquia es un caso que sirve como ejemplo: a pesar de ser la región de Colombia como mayor nivel de información florística (Arbeláez-Cortés ,2013) y de contar con estudios que reportan una riqueza de especies de plantas vasculares de las más altas del mundo (ca. 8000 especies en $66000 \mathrm{~km}^{2}$ ), son evidentes los vacíos de información (Idarraga et al., 2011), en particular, los inventarios florísticos de regiones como el norte y el occidente del departamento son escasos. Ya que estas regiones han sido identificadas recientemente como de alta biodiversidad potencial, elevadas tasas de deforestación y con alta prioridad para la conservación (González-Caro et al., 2015), es necesario llenar los vacíos de información de una manera rápida y eficiente.

Con la revisión de literatura y los sencillos análisis que se presentan en este artículo se muestra que un trabajo intensivo de colecciones botánicas dentro un área tan pequeña como 1 ha puede dar una idea apropiada de la diversidad de plantas a nivel regional, mucho más que los inventarios que se centran en grupos particulares como árboles o plantas leñosas a partir de cierto diámetro, e incluso aportar significativamente a su conocimiento florístico.

\section{CONCLUSIONES}

En el presente estudio se encontraron 318 especies, 82 familias y 173 géneros en una parcela de 1 ha localizadas en el Parque Arví, en un bosque montano del norte de los Andes. Las especies, géneros y familias representan el $47 \%, 48 \%$ y $60 \%$ del total con respecto al Parque Arví. Así mismo, se encontraron 108 especies, 30 géneros y 1 familia no reportada previamente en el Parque Arví. La riqueza de especies/familia en la parcela explica el $89 \%$ de la variación en la riqueza de especies/ familia en al Parque Arví, y la riqueza de géneros, el $75 \%$. Estos resultados indican que un trabajo intensivo de colecciones botánicas dentro de un área tan pequeña como 1 ha permite identificar una porción importante de la diversidad y aportar significativamente al conocimiento de la flora en escalas mayores.

\section{AGRADECIMIENTOS}

Este proyecto fue financiado inicialmente con fondos personales y del Laboratorio de Servicios Ecosistémicos y Cambio Climático (JBMED). Este artículo es un producto del proyecto "Dinámica del Bosque Tropical: Crecimiento y tasas de fijación de carbono en un gradiente ambiental complejo en Colombia", cofinanciado por Colciencias y el Jardín Botánico de Medellín (JBMED), mediante el contrato No. 393 del 2012 y por el Convenio JBMED-Universidad de Leeds 2010.

Especial agradecimiento al señor Darío Cock, por facilitar la reserva para el montaje de la parcela y por la logística prestada. A los biólogos y biólogas quienes colaboraron en la fase de campo. A los especialistas que colaboraron en la determinación del material botánico: R. Callejas, F. J. Roldan, J. Betancur, F. Cardona, F. Alzate, W. Rodríguez, F. Giraldo, J. Pérez. A Lina Vélez por su colaboración en la base de datos, y a Wilson Rengifo por su colaboración en el taller de HUA.

\section{REFERENCIAS BIBLIOGRÁFICAS}

Arbeláez-Cortés, E. (2013). Knowledge of Colombian biodiversity: Published and indexed. Biodiversity and Conservation, 22(12), 2875-2906.

Balslev H., Valencia R., Paz-y-Miño-C G., H. Christensen \& I. Nielsen. (1998). Species Count of Vascular Plants in 1-hectare of humid lowland forest in Amazonian Ecuador. Pp. 584-594. En: Dallmeier, F. \& J.A. Komiskey (eds.): Forest Biodiversity in North, Central and South America, and the Caribbean: Research and Monitoring. Man and the Biosphere Series. (Vol. 21). CRC Press. 792 p. 
Boyle, B., Hopkins, N., Lu, Z., Raygoza Garay, J., Mozzherin, D., Rees, T., Matasci, N., Narro, M., Piel, W., Mckay, S., Lowry, S., Freeland, C., Peet, R. \& Enquist, B. (2013). The taxonomic name resolution service: an online tool for automated standardization of plant names. BMC bioinformatics, 14(1), 16.

Brooks, T.M., Mittermeier R.A., da Fonseca G.A., Gerlach J., Hoffmann M., Lamoreux J.F., Mittermeier C.G., Pilgrim J.D. \& Rodrigues A.S. (2006). Global Biodiversity Conservation Priorities. 313(July), 58-61.

Cayuela, L., De la Cruz, M. \& Ruokolainen, K. (2011). A method to incorporate the effect of taxonomic uncertainty on multivariate analyses of ecological data. Ecography, 34(1), pp.94-102.

Duivenvoorden, J. \& Lips, J. (1995). A land-ecological study of soils, vegetation, and plant diversity in Colombian Amazonia. Wageningen : The Tropenbos Foundation.-III.-(Tropenbos series; 12). 438 p.

Duivenvoorden, J.F. (1994). Vascular plant species counts in the rain forests of the middle Caquetá area, Colombian Amazonia. Biodiversity and Conservation, 3, 685-715.

Duque, A., Álvarez, E., Rodríguez, W. \& Lemos, A. (2013). Impacto de la fragmentación en la diversidad de plantas vasculares en bosques andinos del nororiente de Colombia. Colombia Forestal, 16(2), 115-137.

Espinal, L.S. (2011). Zonas de Vida del Departamento de Antioquia. In A. Idarraga \& R. Callejas, eds. Flora de Antioquia: catálogo de las plantas vasculares. vol. I. Introducción. Medellín, Colombia: Editorial D`Vinni, Bogotá, Colombia, pp. 235-290.

Galeano, G., Suárez, S. \& Balslev, H. (1998). Vascular plant species count in a wet forest in the Chocó area on the Pacific coast of Colombia. Biodiversity \& Conservation, 7, 1563-1575.

Garden, M.B. (2003). Tropicos database. Disponible en: http://www.tropicos.org/ [Consultado el 23 de Noviembre].

Gentry, A.H. (1995). Patterns of diversity and floristic composition in Neotropical montane forests. Biodiversity and conservation of Neotropical montane forests. Proc. symposium, New York Botanical Garden, 1993, 103-126.
Gentry, A.H. (1982). Patterns of neotropical plant-species diversity. Evolutionary Biology, 15, pp.1-84.

Gentry, A.H. \& Dodson, C. (1987). Contribution of no trees to species richness of a tropical rain forest. Biotropica, 19, 149-156.

González-Caro, S., Álvarez, E., Botero, S., Restrepo, Z., Ortiz, C.E. (2015). Antioquia un territorio para conservar. Convenio 4600000674 Gobernación de Antioquia-Jardín Botánico de Medellín 1st ed., Medellín, Colombia: Fondo Editorial Jardín Botánico de Medellín. 32 p.

Gordon, J.E. \& Newton, A.C. (2006). Efficient floristic inventory for the assessment of tropical tree diversity: A comparative test of four alternative approaches. Forest Ecology and Management, 237, 564-573.

Higgins, M. A. \& Ruokolainen, K. (2004). Rapid tropical forest inventory: A comparison of techniques based on inventory data from western Amazonia. Conservation Biology, 18(3), 799-811.

Holdridge, L.R. (1982). Ecología basada en zonas de vida. instituto Interamericano de Cooperación Agrícola (IICA). Colección libros y materiales educativos no. 83, San José, Costa Rica. 216 p.

Idarraga, A., Ortiz, R. \& Callejas, R. (2011). Flora de Antioquia: catálogo de las plantas vasculares. vol. II. Listado de las plantas vasculares del departamento de Antioquia. Programa Expedición Antioquia- 2103. Series Biodiversidad y Recursos Naturales. Universidad de Antioquia, Missouri Botanical. 944 p.

The International Plant Names Index -IPNI-. (2012). The International Plant Names Index. Disponible en: http://www.ipni.org/ [Consultado el 14 de Noviembre, 2014].

Jayakumar, S., Kim, S.S. \& Heo, J. (2011). Floristic inventory and diversity assessment-a critical review. Proceedings of the International Academy of Ecology and Environmental Sciences, 1, 151-168.

Jorgensen, P., Herzog, S., Martínez S., \& Tiessen, H. (2012). Patrones regionales de diversidad y endemismo en las plantas vasculares. En Cambio Climático y Biodiversidad en los Andes Tropicales. p. 428.

Kelly, A.D.L., Tanner, E. V. J., Nic Lughadha, E. M. \& Kapos, V. (1994). Floristics and Biogeography of 
a Rain Forest in the Venezuelan Andes. Journal of Biogeography. 21(4), 421-440.

Kier, G., Kreft, H., Ming Lee, T., Jetz, W., Ibisch, P., Nowicki, C., Mutke, J. \& Barthlott, W. (2009). A global assessment of endemism and species richness across island and mainland regions. Proceedings of the National Academy of Sciences of the United States of America, 106(23), 9322-9327.

Linares-Palomino, R., Cardona, V., Henning, E., Hensen, I., Hoffman, D., Lendzion, J., Soto, D., Herzog, S.K. \& Kessler, M. (2009). Non-woody life-form contribution to vascular plant species richness in a tropical American forest. Forest Ecology: Recent Advances in Plant Ecology, pp.87-99.

Londoño, A.C., Álvarez, E., Forero, E. \& Morton, C. (1995). A New Genus and Species of Dipterocarpaceae from the Neotropics. I. Introduction, Taxonomy. Ecology and Distribution. Brittonia, 47(3), 225-236.

Lópes, W. \& Duque, a J. (2010). Beta diversity in neotropical mountain forests. Caldasia, 32(1), 175-189.

Madriñán, S., Cortés, A.J. \& Richardson, J.E. (2013). Páramo is the world's fastest evolving and coolest biodiversity hotspot. Frontiers in Genetics, 4(October), 1-7.

Myers, N., Mittermeler, R., Mittermeler, C., da Fonseca, G.A.b. \& Kent, J. (2000). Biodiversity hotspots for conservation priorities. Nature, 403(February), pp.853-8.

Natura, F. \& Holos, F., 2001. Plan Maestro Parque Regional Arví. Tomo I. Corporación Autónoma Regional del Centro de Antioquia. Medellín, Colombia. $360 \mathrm{p}$.

Phillips, O.L., Vásquez, R., Nuñez, P., Monteagudo, A.B., Chuspe, M.E., Galiano, W., Peña, A., Timaná, M., Yli-Halla, M. \& Rose, S. (2003). Efficient plot-based floristic assessment of tropical forests. Journal of tropical ecology, 19 (6), 629-645.

Ruokolainen, K., Tuomisto, H., Macía, M.J., Higgins, M.A. \& Yli-Halla, M. (2007). Are floristic and edaphic patterns in Amazonian rain forests congruent for trees, pteridophytes and Melastomataceae?. Journal of Tropical Ecology, 23(01), 13-25.

Ter Steege, H., Sabatier, D., Castellanos, H., Van Andel, T., Duivenvoorden, J., De Oliverira, A., Ek, R., Lilwah, R., Maas, P. \& Mori, S. (2000). An analysis of the floristic composition and diversity of Amazonian forests including those of the Guiana Shield. Journal of Tropical Ecology, 16(6), 801-828.

Tchouto, M.G.P., De Boer, W.F., De Wilde, J.J.F. \& Van der Maesen, L.J.G. (2006). Diversity patterns in the flora of the Campo-Ma'an rain forest, Cameroon: Do tree species tell it all?. Biodiversity and Conservation, 15, 1353-1374.

Toro, J.L. (2000). Árboles y arbustos del parque regional Arvi. Medellín: Corporación Autónoma Regional del Centro de Antioquia. 281 p.

Toro, J.L. (2009). Estado del conocimiento de la Flora Silvestre en la jurisdicción de Corantioquia., Medellín, Colombia. 468 p.

The Plant List -TPL-. (2010). The Plant List. Version 1. Disponible en: http://www.theplantlist.org/ [Consultado el 20 de Abril, 2014].

Valencia, R., Balslev, H. \& Paz y Miño, G. (1994). High tree alpha-diversity in Amazonian Ecuador. Biodiversity and Conservation, 3(1), 21-28.

Vélez-Arango, G. \& Cogollo-Pacheco, A. (2007). Primer registro del género Sapranthus (Annonaceae) y una nueva especie para Suramérica. , 29(2), 229-233.

Whitmore, T.C., Peralta, R. \& Brown, K. (1985). Total species count in a Costa Rican rain forest. Journal of Tropical Ecology, 1(4), 375-378.

Williams-Linera, G., Palacios-Rios, M. \& Hernández-Gómez, R. (2005). Fern richness, tree species surrogacy, and fragment complementarity in a Mexican tropical montane cloud forest. Biodiversity and Conservation, 14(1), 119-133.

Young, K.R., Ulloa, C., Luteyn, J. \& Knapp, S. (2002). Plant Evolution and Endemism in Andean South America: An Introduction. The Botanical Review, 68(1), 4-21. 


\section{ANEXO 1.}

Listado de especies de plantas vasculares encontradas en una parcela de 1 ha en la Reserva Montevivo. Entre paréntesis se presenta, separados por coma: A) el número de colección correspondiente al código del autor principal HDH. B) El hábito de crecimiento de la especie de acuerdo con las categorías presentadas en la metodología, y C) si la especie se encontró $(\mathrm{S})$ o no $(\mathrm{N})$ en la colección del Parque Arví; el código Na significa que no aplica, cuando la muestra botánica no fue identificada hasta especie. Copias de los excicados se encuentran en los Herbarios de la Universidad de Antioquia (HUA) y el Jardín Botánico de Medellín (JAUM).

\section{MAGNOLIOPHYTA}

ACANTHACEAE: Aphelandra runcinata (202, Ab, S); Blechum pyramidatum (201, Ab, N); ACTINIDIACEAE: Saurauia ursina (203, Ab, S); AMARYLLIDACEAE: Bomarea bredemeyerana (403, Eh, N); Bomarea diffracta (402, Eh, N); Bomarea patinii (404, Eh, S); ANACARDIACEAE: Mauria heterophyIla (205, Ar, S); Mauria sp (204, Ab, Na); APOCYNACEAE: Blepharodon grandiflorum (218, Eh, N); Cynanchum sp1 (216, Eh, Na); Cynanchum sp2 (217, Eh, Na); AQUIFOLIACEAE: Ilex danielis (206, Ab, S); Ilex laurina (207, Ar, S); Ilex nervosa (208, Ar, S); ARACEAE: Anthurium caucanum (396, Ht, S); Anthurium cupreum (395, Ht, S); Anthurium longegeniculatum (399, Ht, S); Anthurium microspadix (397, Ht, S); Anthurium nigrescens (394, Ep, S); Anthurium scandens (400, Ht, S); Anthurium sec. belolonchium (398, Ht, Na); Anthurium sp (401, Ht, $\mathrm{Na})$; ARALIACEAE: Dendropanax caucanus (215, $\mathrm{Ar}, \mathrm{N})$; Dendropanax sp1 (214, Ab, Na); Oreopanax floribundus (212, Ar, S); Oreopanax sp1 (213, Ab, $\mathrm{Na})$; Schefflera quinduensis (209, Li, N); Schefflera sphaerocoma (211, Ar, S); Schefflera trianae (210, Ar, S); ASTERACEAE: Ageratina popayanensis (223, Ab, S); Baccharis oblongifolia (234, Ab, S); Calea angosturana (235, Eh, N); Dendrophorbium barkleyanum (233, Eh, N); Jungia ferruginea (222, Ht, N);
Mikania aschersonii (232, Eh, N); Mikania banisteriae (221, Eh, S); Mikania clematidiflora (219, Eh, N); Mikania fragrans (230, Eh, N); Mikania sp1 (220, Eh, $\mathrm{Na})$; Mikania sp2 (228, Eh, Na); Mikania sylvatica (229, Eh, S); Munnozia senecionidis (227, Eh, S); Oligactis volubilis (236, Eh, S); Pentacalia sonsonensis (224, Eh, N); Pentacalia sp1 (231, Eh, Na); Pentacalia supernitens (226, Eh, N); Pentacalia trianae (225, Eh, N); BALANOPHORACEAE: Corynaea crassa (239, Ht, N); BORAGINACEAE: Tournefortia fuliginosa (238, Ab, S); Varronia curassavica (237, Ar, N); BROMELIACEAE: Guzmania nidularioides (408, Ep, N); Guzmania triangularis (407, Ep, S); Mezobromelia capituligera (410, Ep, N); Pitcairnia pectinata (409, Ht, N); Racinaea contorta (411, Ep, $\mathrm{N})$; Racinaea subalata (412, Ep, S); Tillandsia biflora (413, Ep, N); Tillandsia confinis (406, Ep, N); Tillandsia truncata (405, Ep, N); BRUNELLIACEAE: BruneIlia trianae (240, Ar, S); CAMPANULACEAE: Burmeistera asclepiadea (244, Ab, N); Burmeistera glabrata (243, Ab, S); Centropogon ferrugineus (242, Eh, S); Centropogon solanifolius (241, Ab, N); CAPRIFOLIACEAE: Valeriana clematitis (387, Eh, S); Valeriana sp (388, Ep, Na); Viburnum anabaptista (245, Ar, S); Viburnum pichinchense (247, Ar, N); Viburnum undulatum (246, Ar, N); CELASTRACEAE: Celastrus liebmannii (248, Li, S); Maytenus sp (249, Ab, Na); CHLORANTHACEAE: Hedyosmum bonplandianum (250, Ar, S); Hedyosmum sp (251, Ar, Na); CHRYSOBALANACEAE: Couepia sp (252, Ab, Na); CLETHRACEAE: Clethra fagifolia (253, Ar, S); Clethra revoluta (254, Ar, S); CLUSIACEAE: Chrysochlamys colombiana (257, Ar, S); Clusia ducu (258, Ar, N); Clusia ducuoides (260, Ar, S); Clusia multiflora (259, Ar, S); Clusia sp (261, Ep, Na); Vismia baccifera (255, Ar, S); Vismia laevis (256, Ar, S); CUNONIACEAE: Weinmannia balbisiana (262, Ar, S); Weinmannia pubescens (263, Ar, S); Weinmannia sp (264, Ab, Na); CYPERACEAE: Rhynchospora hieronymi (415, Ht, N); Rhynchospora locuples (416, Ht, N); Rhynchospora macrochaeta (417, Ht, $\mathrm{N})$; Rhynchospora sp (418, Ht, Na); Uncinia hamata (414, Ht, S); DICHAPETALACEAE: Stephanopodium aptotum (265, Ab, S); DIOSCOREACEAE: 
Dioscorea piperifolia (419, Eh, N); ERICACEAE: Bejaria aestuans (266, Ab, N); Cavendishia bracteata (271, Li, S); Cavendishia pubescens (272, Li, S); Cavendishia tarapotana (267, Li, N); Gaultheria buxifolia (275, Ab, S); Gaultheria erecta (273, Ab, S); Gaultheria rigida (274, Ab, S); Psammisia pennellii (269, Li, S); Psammisia sodiroi (268, Ab, S); Sphyrospermum buxifolium (277, Ep, N); Thibaudia floribunda (270, Li, N); Vaccinium corymbodendron (276, Ab, S); ESCALLONIACEAE: Escallonia paniculata (290, Ar, S); EUPHORBIACEAE: Alchornea acutifolia (280, Ar, S); Alchornea triplinervia (282, Ar, $\mathrm{N})$; Alchornea verticilata (281, Ar, N); Croton magdalenensis (279, Ar, S); Hieronyma antioquensis (283, Ar, S); Sapium stylare (284, Ar, S); Tetrorchidium gorgonae (278, Ab, N); GENTIANACEAE: Macrocarpaea macrophylla (286, Ab, S); GESNERIACEAE: Columnea dimidiata (288, Ab, N); Glossoloma ichthyoderma (287, Ab, N); Kohleria affinis (289, Ep, S); HYDRANGEACEAE: Hydrangea peruviana (291, Li, S); LACISTEMATACEAE: Lozania mutisiana (293, Ar, S); LAMIACEAE: Aegiphila sp (389, Ab, Na); LAURACEAE: Cinnamomum triplinerve (294, Ar, N); Nectandra laurel (298, Ab, S); Nectandra reticulata (297, Ab, N); Ocotea costulata (296, Ab, S); Ocotea sp (301, Ab, Na); Persea chrysophylla (299, Ab, S); Persea sp (300, Ar, Na); Pleurothyrium sp (295, Ab, Na); LECYTHIDACEAE: Eschweilera antioquensis (497, Ab, S); LORANTHACEAE: Cladocolea archeri (498, Ht, S); MALPIGHIACEAE: Stigmaphyllon bogotense (499, Li, S); Stigmaphyllon sp (500, Li, Na); MELASTOMATACEAE: Axinaea macrophylla (505, Ar, S); Blakea quadrangularis (504, Li, S); Leandra sp (514, Ab, $\mathrm{Na})$; Meriania antioquiensis (506, Ab, S); Meriania nobilis (502, Ar, S); Miconia caudata (508, Ab, S); Miconia dolichorrhyncha (509, Ab, N); Miconia jahnii (512, Ar, N); Miconia lehmannii (510, Ab, S); Miconia psychrophila (507, Ab, N); Miconia theaezans (501, Ar, S); Miconia wurdackii (513, Ar, N); Monolena sp (511, Ep, Na); Tibouchina lepidota (503, Ar, S); Tibouchina sp (515, Ab, Na); MELIACEAE: Ruagea glabra (516, Ar, S); MONIMIACEAE: Siparuna laurifolia (517, Ab, N); Siparuna stellulata (518, Li,
S); MORACEAE: Ficus mutisii (519, Ab, N); MYRICACEAE: Morella pubescens (520, Ar, S); MYRTACEAE: Eugenia sp (313, Ab, Na); Myrcia popayanensis (309, Ar, S); Myrcia splendens (308, Ar, S); Myrcianthes rhopaloides (310, Ar, S); Myrtaceae sp1 (629, $\mathrm{Ab}, \mathrm{Na})$; Psidium arayan (311, Ar, N); Psidium sp (312, Ab, Na); ORCHIDACEAE: Cleistes sp (438, Ht, $\mathrm{Na})$; Elleanthus aurantiacus (429, Ht, S); Epidendrum cylindrostachys (428, Ep, N); Epidendrum decurviflorum (426, Ep, N); Epidendrum paniculatum (427, Ep, S); Epidendrum secundum (425, Ep, N); Epidendrum sophronitoides (424, Ep, N); Epidendrum sp (423, Ep, Na); Gomphichis sp1 (431, Ht, $\mathrm{Na})$; Gomphichis sp2 (432, Ht, Na); Habenaria sp (444, Ht, Na); Malaxis parthonii (430, Ht, N); MaxiIlaria aurea (441, Ht, S); Maxillaria sp (440, Ep, Na); Oncidium cocciferum (437, Ep, S); Platystele sp (439, Ep, Na); Pleurothallis sp1 (445, Ep, Na); Pleurothallis sp2 (446, Ht, Na); Pleurothallis sp3 (436, Ep, Na); Pleurothallis tuerckheimii (435, Ep, N); Prescottia sp1 (443, Ht, Na); Prescottia sp2 (442, Ht, $\mathrm{Na})$; Stelis crassilabia (433, Ht, N); Stelis velutina (434, Ep, N); PASSIFLORACEAE: Passiflora alnifolia (316, Eh, S); Passiflora apoda (317, Eh, S); PICRAMNIACEAE: Picramnia gracilis (369, Ab, S); PIPERACEAE: Peperomia acuminata (318, Ht, S); Peperomia colorata (323, Ep, N); Peperomia crotalophora (329, Ht, N); Peperomia tetraphylla (322, Ep, S); Peperomia trianae (321, Ep, N); Peperomia trinervula (319, Ep, N); Peperomia truncivaga (320, Ep, N); Peperomia zipaquirana (330, Ep, N); Piper adscendens (331, Ht, N); Piper antioquiense (328, Ab, N); Piper archeri (324, Ab, N); Piper artanthe (326, Ab, S); Piper calceolarium (325, Ab, S); Piper daniel-gonzalezii (333, Ht, S); Piper lacunosum (327, Ab, S); Piper sp (332, Ab, Na); POACEAE: Festuca sp (422, Ht, $\mathrm{Na})$; POLYGALACEAE: Monnina celastroides (334, Ab, N); Monnina philippiana (337, Ab, S); Monnina solandraefolia (335, Eh, N); Monnina speciosa (336, Li, N); POLYGONACEAE: Muehlenbeckia tamnifolia (338, Li, S); PRIMULACEAE: Cybianthus pastensis (306, Ab, S); Geissanthus occidentalis (305, Ar, S); Myrsine coriacea (303, Ar, N); Stylogine sp (307, $\mathrm{Ab}, \mathrm{Na})$; Stylogyne turbacensis (302, $\mathrm{Ab}, \mathrm{N})$; 
Geissanthus sp (304, Ar, Na); PROTEACEAE: Panopsis yolombo (340, Ar, S); Roupala monosperma (339, Ar, S); RANUNCULACEAE: Clematis sp (341, Li, Na); RHAMNACEAE: Rhamnus goudotiana (342, Ab, S); ROSACEAE: Prunus integrifolia (345, Ar, S); Prunus opaca (344, Ab, N); Prunus sp (343, Ab, Na); Rubus floribundus (347, Eh, N); Rubus roseus (346, Eh, N); RUBIACEAE: Coccocypselum lanceolatum (353, Ht, S); Faramea oblongifolia (357, Ab, S); Galium hypocarpium (354, Eh, N); Hoffmannia sp (359, Ab, Na); Ladenbergia macrocarpa (356, Ab, S); Manettia cordifolia (365, Eh, N); Manettia corticifera (349, Eh, N); Nertera granadensis (350, Ht, S); Palicourea andaluciana (358, Ab, S); Palicourea angustifolia (363, Ab, S); Palicourea garciae (348, Ab, S); Palicourea lyristipula (364, Ab, S); Palicourea sp (362, Ab, Na); Psychotria aubletiana (351, Ab, S); Psychotria graciliflora (352, Ab, N); Psychotria sp1 (360, Ab, Na); Psychotria sp2 (361, Ab, Na); Spermacoce remota (355, Ht, N); RUTACEAE: Zanthoxylum melanostictum (366, Ar, S); Zanthoxylum quinduense (367, Ar, N); SALICACEAE: Casearia quinduensis (285, Ab, N); SAPINDACEAE: Allophylus excelsus (368, Ar, N); Billia rosea (292, Ar, N); SMILACACEAE: Smilax sp1 (421, Li, Na); Smilax sp2 (420, Li, Na); SOLANACEAE: Cestrum sp (379, Li, $\mathrm{Na})$; Lycianthes acutifolia (378, Ab, N); Lycianthes $\mathrm{sp}$ (380, Li, Na); Markea sp (376, Ab, Na); Schultesianthus coriaceus (377, Li, S); Solanum aphyodendron (375, Ab, S); Solanum aturense (371, Li, N); Solanum dichroandrum (745, Li, S); Solanum dolosum (374, Ab, S); Solanum nutans (370, Ab, N); STAPHYLEACEAE: Turpinia occidentalis (381, Ar, S); STYRACACEAE: Styrax sp (382, Ab, Na); THEACEAE: Freziera arbutifolia (383, Ab, S); Ternstroemia macrocarpa (384, Ar, N); THYMELAEACEAE: Daphnopsis sp (385, Ab, Na); URTICACEAE: Pilea pubescens (386, Ht, N); VERBENACEAE: Citharexylum sp (390, Ar, Na); VIOLACEAE: Viola stipularis (391, Ht, S); VITACEAE: Cissus trianae (392, Li, S); WINTERACEAE: Drimys granadensis (393, Ar, S).

\section{PTERIDOPHYTA}

ASPLENIACEAE: Asplenium auritum (451, Ep, S); Asplenium pteropus (452, Ep, N); ATHYRIACEAE: Diplazium sp1 (467, Ht, Na); Diplazium sp2 (468, $\mathrm{Ht}, \mathrm{Na}$ ); BLECHNACEAE: Blechnum cordatum (455, Ht, S); Blechnum fragile (453, Ep, N); Blechnum stipitellatum (454, Ht, S); CYATHEACEAE: Cyathea caracasana (457, Ar, S); Cyathea fulva (456, Ar, N); Cyathea meridensis (458, Ar, N); DENNSTAEDTIACEAE: Paesia glandulosa (459, Ht, S); Pteridium arachnoideum (460, Ht, S); DRYOPTERIDACEAE: Arachniodes denticulata (469, Ht, S); Elaphoglossum antioquianum (464, Ep, S); Elaphoglossum latifolium (462, Ht, S); Elaphoglossum luridum (461, Ep, N); Elaphoglossum muscosum (465, Ep, N); Elaphoglossum Sec. Lepiglossa (466, Ep, Na); Elaphoglossum sp1 (470, Ep, Na); Elaphoglossum sp2 (471, Ep, Na); Elaphoglossum sp3 (472, Ht, Na); Elaphoglossum urophyllum (463, Ht, N); EQUISETACEAE: Equisetum bogotense (447, Ht, S); GLEICHENIACEAE: Sticherus rubiginosus (473, Ht, S); HYMENOPHYLLACEAE: Hymenophyllum farallonense (475, Ep, N); Hymenophyllum fucoides (476, Ep, N); Hymenophyllum interruptum (477, Ep, N); LYCOPODIACEAE: Lycopodium clavatum (449, Ht, S); Lycopodium jussiaei (450, Ht, S); Lycopodium thyoides $(448, \mathrm{Ht}, \mathrm{S})$; MARATTIACEAE: Marattia laevis (478, Ht, N); Marattia sp (479, Ht, Na); POLYPODIACEAE: Campyloneurum mphostenon (480, Ep, N); Cochlidium serrulatum (489, Ep, S); Grammitis limbata (474, Ep, N); Lellingeria phlegmaria (490, Ep, $\mathrm{N})$; Melpomene pilosissima (488, Ep, N); Pleopeltis macrocarpa (481, Ep, N); Polypodium fraxinifolium (483, Ep, S); Polypodium levigatum (486, Ep, N); Polypodium sp (482, Ep, Na); Serpocaulon funckii (485, Ep, S); Serpocaulon semipinnatifidum (484, Ep, S); Terpsichore taxifolia (487, Ep, N); PTERIDACEAE: Eriosorus flexuosus (491, Ht, S); Eriosorus glaberrimus (492, Eh, N); Eriosorus velleus (493, Ht, N); THELYPTERIDACEAE: Thelypteris deflexa (495, Ht, N); Thelypteris hispidula (494, Ht, N).

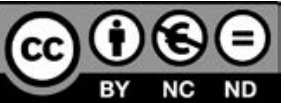

